\title{
PROPOSTA PARA A CRIAÇÃO DE JOGOS EDUCATIVOS DIGITAIS
}

\section{PROPOSAL FOR THE CREATION OF DIGITAL EDUCATIONAL GAMES}

\author{
Rafael Feyh Jappur. Dr. ${ }^{1}$ \\ Fernando José Spanhol. Dr. ${ }^{2}$ \\ (1) Faculdade de Tecnologia Senac Florianópolis \\ rjappur@gmail.com \\ (2) Universidade Federal de Santa Catarina-Campos Araranguá \\ profspanhol@gmail.com
}

Jogos educativos digitais, processo de ensino e aprendizagem, propostas pedagógica e lúdica.

Os jogos educativos digitais têm o potencial de agregar valor ao processo de ensino e aprendizagem de conteúdos em sala de aula. Contudo, os professores ou mediadores desse processo enfrentam dificuldades em encontrar e utilizar bons jogos como ferramenta de aprendizagem. Nesse sentido, este trabalho visa apresentar uma proposta pedagógica e lúdica para a criação de jogos educativos digitais, no intuito acrescentar conhecimento aos desenvolvedores para que produzam bons jogos. Os procedimentos metodológicos utilizados foram da pesquisa bibliográfica, composta por pesquisas exploratórias e bibliométricas. Com referência aos resultados desta pesquisa propõem-se, como proposta pedagógica, a utilização da taxionomia de Bloom revisada e os critérios para aprendizagem mediada de Feuerstein; e uso de quatro elementos para a proposta lúdica: mecânica, estética, história/narrativa, e tecnologia.

Digital educational games, teaching and learning process, pedagogical and ludic proposals.

Digital educational games have the potential to add value to the process of teaching and learning content in the classroom. However, teachers or mediators in this process face difficulties in finding and using good games as a learning tool. In this sense, this paper aims to present a pedagogical and ludic proposal for the creation of digital educational games, in order to increase the knowledge to the developers to produce good games. The methodological procedures used were the bibliographical research, composed of exploratory and bibliometric researches. With reference to the results of this research, we propose, as pedagogical proposal, the use of the revised Bloom taxonomy and the criteria for Feuerstein's mediated learning; And the application of four elements for the ludic proposal: mechanics, aesthetics, history / narrative, and technology. 


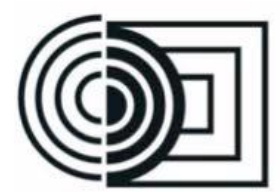

\section{Introdução}

Os jogos educativos digitais são uma estratégia pedagógica ativa para o ensino e aprendizagem de conteúdos em sala de aula. Todavia, constata-se - conforme apresentam Kirriemuir e Mcfarlane (2004); Balasubramanian e Wilson, (2006), Echeverría et al. (2011), entre outros - que os professores ou mediadores do processo de aprendizagem possuem dificuldades para aplicar os jogos em sala de aula.

Estas dificuldades, conforme Balasubramanian e Wilson (2006), repousam na baixa utilização dos jogos educativos digitais nas escolas e, para muitos educadores, o desafio é encontrar e utilizar bons jogos como ferramenta de aprendizagem. Para os autores, os jogos digitais, no seu processo de criação têm sido usados de forma não articulada aos princípios e necessidades pedagógicas. Isto muitas vezes gera fragilidades ou dificuldades no que tange à seleção e aplicação pelos educadores, por não compreenderem o valor agregado à aprendizagem.

\section{Metodologia}

A natureza desta pesquisa possui mais similaridades com a pesquisa básica; quanto à forma de abordagem, representa uma pesquisa qualitativa; quanto aos objetivos, situa-se em três categorias: exploratória, descritiva e explicativa. Em relação aos procedimentos adotados para a coleta de dados, foi composta preponderantemente da pesquisa bibliográfica (pesquisas bibliométricas e exploratórias).

\section{Os jogos educativos digitais}

A produção científica sobre jogos educacionais em mídias digitais vem crescendo nas últimas duas décadas, com pesquisas sobre o potencial desses jogos para o processo de ensino e aprendizagem (PRENSKY, 2001; MORENOGER et al., 2008; ECHEVERRÍA et al., 2011, $16^{\circ}$ Ergodesign - Congresso Internacional de Ergonomia e Usabilidade de Interfaces Humano Tecnológica: Produto, Informações Ambientes Construídos e Transporte

$16^{\circ}$ USIHC - Congresso Internacional de Ergonomia e Usabilidade de Interfaces Humano Computador

CINAHPA | 2017 - Congresso Internacional de Ambientes Hipermídia para Aprendizagem.

entre outros). Dentro dessa amplitude, autores como Prensky (2001) afirmam que um projeto de jogo educacional eficaz deve alcançar um equilíbrio entre a diversão e valor educacional, sendo que o sucesso desse equilíbrio se alcança por meio da aplicação de um modelo de design para o jogo.

O uso de jogos digitais como ferramenta educacional está lentamente se tornando uma prática aceita em ambientes de aprendizagem (ECHEVERRÍA et al., 2011). Esses jogos apresentam diversas possibilidades para o desenvolvimento do conhecimento e também ajudam a melhorar o processo de ensino e aprendizagem nas escolas (VILLALTA et al., 2011). O uso de jogos em sala de aula tem provado ser eficaz para a motivação, colaboração e para os resultados da aprendizagem.

Savi (2011) salienta, em sua pesquisa, que, para que um jogo educativo possa ter qualidade, é necessário que esteja bem definido, que motive os usuários a jogar e que promova a aprendizagem de conteúdos por meio de atividades divertidas, prazerosas e desafiadoras.

\section{Modelos de jogos educativos digitais}

Com base na revisão bibliográfica e no estudo mais aprofundado dos modelos pesquisados, constatou-se um consenso entre os autores, segundo o qual, para um bom jogo educativo digital é importante que haja uma proposta educacional e lúdica.

Conforme apresentam Echeverría et al. (2011) e Villalta et al. (2011), a dimensão lúdica refere-se aos elementos do jogo, respeitando, todavia, as questões impostas pela dimensão educativa ou pedagógica. Aleven et al., (2010) afirmam que um bom jogo educativo digital deve possuir um projeto pedagógico e um projeto de jogo divertido.

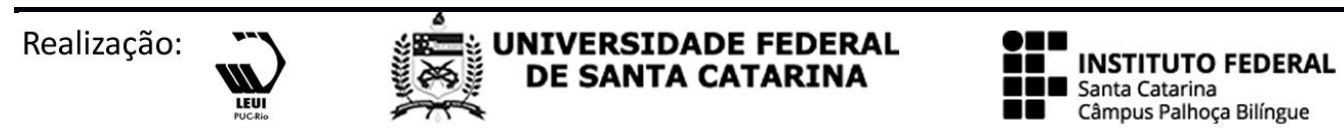




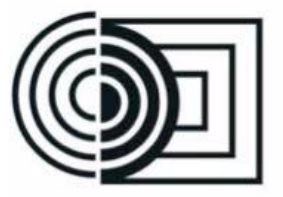
CINAHPA

Nesse sentido, elencaram-se, no quadro 01, as propostas pedagógicas e lúdicas, apresentadas pelos autores dos modelos identificados e selecionados.

\begin{tabular}{|c|c|c|}
\hline $\begin{array}{c}\text { Modelos } \\
\text { Conceituais }\end{array}$ & $\begin{array}{c}\text { Propostas } \\
\text { Pedagógicas }\end{array}$ & $\begin{array}{l}\text { Propostas } \\
\text { Lúdicas }\end{array}$ \\
\hline $\begin{array}{l}\text { Mayer } \\
(2002)\end{array}$ & $\begin{array}{l}\text { Taxionomia de } \\
\text { Bloom Revisada } \\
\text { (ANDERSON et } \\
\text { al., 2001) }\end{array}$ & Não apresenta. \\
\hline $\begin{array}{l}\text { Echeverría } \\
\text { et al. (2011) }\end{array}$ & $\begin{array}{l}\text { Taxionomia de } \\
\text { Bloom revisada } \\
\text { (ANDERSON et } \\
\text { al., 2001). }\end{array}$ & $\begin{array}{l}\text { Mecânica, história, } \\
\text { estética, tecnologia } \\
\text { (SCHELL, 2008). }\end{array}$ \\
\hline $\begin{array}{l}\text { Aleven et al. } \\
(2010)\end{array}$ & $\begin{array}{l}\text { Taxionomia de } \\
\text { Bloom revisada / } \\
\text { Princípios do } \\
\text { design instrucional } \\
\text { (ANDERSON et } \\
\text { al., 1995; } \\
\text { MAYER; } \\
\text { MORENO, 2003; } \\
\text { GEE, 2007). }\end{array}$ & $\begin{array}{l}\text { Mecânica, } \\
\text { dinâmica, estética } \\
\text { (HUNICKE et al., } \\
\text { 2004). }\end{array}$ \\
\hline $\begin{array}{l}\text { Villalta et } \\
\text { al. (2011) }\end{array}$ & $\begin{array}{l}\text { Não apresentam } \\
\text { proposta única, } \\
\text { mas devem estar } \\
\text { alinhadas com as } \\
\text { estratégias } \\
\text { instrucionais. }\end{array}$ & $\begin{array}{l}\text { Apresenta um guia } \\
\text { de avaliação, que } \\
\text { inclui: mecânica, } \\
\text { progressão, } \\
\text { metodologia, } \\
\text { colaboração, } \\
\text { informação na tela, } \\
\text { holismo. }\end{array}$ \\
\hline Savi (2011) & $\begin{array}{l}\text { Três primeiros } \\
\text { níveis da } \\
\text { taxionomia de } \\
\text { Bloom } \\
\text { (conhecimento, } \\
\text { compreensão e } \\
\text { aplicação) } \\
\text { (BLOOM et al., } \\
\text { 1977); } \\
\text { aprendizagem de } \\
\text { curto e longo prazo } \\
\text { (MOODY; } \\
\text { SINDRE, 2003). }\end{array}$ & $\begin{array}{l}\text { Percepção dos } \\
\text { alunos - Nível } 1 \\
\text { (KIRKPATRICK, } \\
\text { 1994); Modelo A, } \\
\text { R, C, S - Atenção, } \\
\text { relevância, } \\
\text { competência e } \\
\text { satisfação } \\
\text { (KELLER, 1987); } \\
\text { Imersão, interação } \\
\text { social, desafio, } \\
\text { divertimento e } \\
\text { competência } \\
\text { (SWEETSER; } \\
\text { WYETH, 2005; } \\
\text { POELS; KORT; } \\
\text { IJSSELSTEIJN, } \\
\text { 2007; GÁMEZ, } \\
\text { 2009; } \\
\text { TAKATALO et } \\
\text { al., 2010). }\end{array}$ \\
\hline $\begin{array}{l}\text { Klein et al. } \\
(2000)\end{array}$ & $\begin{array}{l}\text { Feuerstein - Seis } \\
\text { critérios de } \\
\text { mediação: } \\
\text { intencionalidade e } \\
\text { reciprocidade, } \\
\text { transcendência, } \\
\text { significado, } \\
\text { competência e } \\
\text { autorregulação }\end{array}$ & Não apresenta. \\
\hline
\end{tabular}

$16^{\circ}$ Ergodesign - Congresso Internacional de Ergonomia e Usabilidade de Interfaces Humano Tecnológica: Produto, Informações Ambientes Construídos e Transporte

$16^{\circ}$ USIHC - Congresso Internacional de Ergonomia e Usabilidade de Interfaces Humano Computador

CINAHPA | 2017 - Congresso Internacional de Ambientes Hipermídia para Aprendizagem.

\begin{tabular}{|l|l|l|}
\hline & $\begin{array}{l}\text { (FEUERSTEIN, } \\
\text { 1980; KLEIN, } \\
\text { 1996). }\end{array}$ & \\
& $\begin{array}{l}\text { Feuerstein - Três } \\
\text { critérios de } \\
\text { mediação: } \\
\text { intencionalidade e } \\
\text { Campos e } \\
\text { Macedo } \\
\text { reciprocidade, } \\
\text { transcendência, } \\
\text { significado } \\
\text { (FEUERSTEIN, } \\
\text { 1980). }\end{array}$ & Não apresenta. \\
\hline $\begin{array}{l}\text { Mediação-todos } \\
\text { os critérios de } \\
\text { mediação (12 } \\
\text { critérios); } \\
\text { processo } \\
\text { cognitivo; } \\
\text { mecanismos de } \\
\text { aprendizagem } \\
\text { (FEUERSTEIN, } \\
\text { 1980). }\end{array}$ & Não apresenta. \\
\hline \multicolumn{2}{|l|}{$\begin{array}{l}\text { Quadro } \\
\text { Fonte: Jappur (2014). }\end{array}$} \\
\hline
\end{tabular}

No quadro 2, apresenta-se uma síntese das propostas pedagógicas em conformidade com os autores consultados.

\begin{tabular}{|c|c|}
\hline Propostas Pedagógicas & Fontes \\
\hline $\begin{array}{c}\text { Três primeiros níveis da } \\
\text { Taxionomia de Bloom } \\
\text { Original }\end{array}$ & $\begin{array}{l}\text { Bloom et al. (1977); SAVI } \\
\text { (2011). }\end{array}$ \\
\hline $\begin{array}{c}\text { Taxionomia de Bloom } \\
\text { Revisada }\end{array}$ & $\begin{array}{l}\text { Anderson et al. (2001); } \\
\text { Mayer (2002); Aleven et } \\
\text { al. (2010); Echeverría et al. } \\
\text { (2011). }\end{array}$ \\
\hline $\begin{array}{l}\text { Princípios do design } \\
\text { instrucional }\end{array}$ & $\begin{array}{c}\text { Anderson et al. (1995); } \\
\text { Mayer e Moreno (2003); } \\
\text { Gee (2007); Aleven et al. } \\
\text { (2010); Villalta et al. } \\
\text { (2011). }\end{array}$ \\
\hline $\begin{array}{l}\text { Aprendizagem de curto e } \\
\text { longo prazo }\end{array}$ & $\begin{array}{l}\text { Moody e Sindre (2003); } \\
\text { Savi (2011). }\end{array}$ \\
\hline $\begin{array}{c}\text { Critérios para a mediação: } \\
\text { três critérios fundamentais } \\
\text { de mediação - } \\
\text { intencionalidade e } \\
\text { reciprocidade, } \\
\text { transcendência, significado; } \\
\text { e os nove outros critérios de } \\
\text { mediação ( } 12 \text { critérios ao } \\
\text { total). }\end{array}$ & $\begin{array}{c}\text { Feuerstein (1980); Klein } \\
\text { (1996); Klein et al. (2000); } \\
\text { Gomes (2001); Campos e } \\
\text { Macedo (2011). }\end{array}$ \\
\hline
\end{tabular}

Quadro 2- Síntese das propostas pedagógicas Fonte: Jappur (2014) 


\section{$16^{\circ}$ \\ ERGODESIGN USIHC CINAHPA}

No quadro 3, apresenta-se uma síntese das propostas lúdicas descritas pelos autores pesquisados.

\begin{tabular}{|l|l|}
\hline Propostas lúdicas & Fontes \\
\hline Mecânica & $\begin{array}{l}\text { Hunicke et al., 2004; Schell, } \\
\text { 2008; Aleven et al., 2010; } \\
\text { Echeverría et al., 2011; Villalta } \\
\text { et al., 2011. }\end{array}$ \\
\hline História/Narrativa & $\begin{array}{l}\text { Schell, 2008; Echeverría et al., } \\
\text { 2011; Villalta et al., 2011. }\end{array}$ \\
\hline Estética & $\begin{array}{l}\text { Hunicke et al., 2004; Schell, } \\
\text { 2008; Aleven et al., 2010; } \\
\text { Echeverría et al., 2011. }\end{array}$ \\
\hline Tecnologia & $\begin{array}{l}\text { Schell, 2008; Echeverría et al., } \\
\text { 2011. }\end{array}$ \\
\hline Dinâmica/progressão & $\begin{array}{l}\text { Hunicke et al., 2004; Aleven et } \\
\text { al., 2010; Villalta et al., 2011. }\end{array}$ \\
\hline Metodologia & Villalta et al., 2011. \\
\hline Colaboração & Villalta et al., 2011. \\
\hline Informação na tela & Villalta et al., 2011. \\
\hline Holismo & Villalta et al., 2011. \\
\hline
\end{tabular}

Quadro 3 - Síntese das propostas lúdicas

Fonte: Fonte: Jappur (2014).

Contudo, verifica-se certo consenso entre os autores, referente aos elementos lúdicos, os quatro primeiros elementos apresentados no quadro 3 (mecânica, história/narrativa, estética e tecnologia), pois são os elementos mais citados pelos autores pesquisados.

\section{Propostas pedagógica e lúdica para a criação de jogos educativos digitais}

\section{Proposta pedagógica para a criação de jogos educativos digitais}

Conforme apresentado, se constata que diferentes abordagens educacionais podem ser seguidas para o projeto pedagógico de desenvolvimento de jogos educativos digitais. Na perspectiva de agregar conhecimento à temática, que neste trabalho propõe-se a utilização de duas propostas pedagógicas para o processo de criação dos jogos: Taxionomia de Bloom revisada; e Critérios para aprendizagem mediada de Feuerstein.

Para a criação dos jogos, os desenvolvedores $16^{\circ}$ Ergodesign - Congresso Internacional de Ergonomia e Usabilidade de Interfaces Humano Tecnológica: Produto, Informações Ambientes Construídos e Transporte

$16^{\circ}$ USIHC - Congresso Internacional de Ergonomia e Usabilidade de Interfaces Humano Computador

CINAHPA | 2017 - Congresso Internacional de Ambientes Hipermídia para Aprendizagem.

geralmente utilizam os princípios e processos do design instrucional, e muitas pesquisas têm sido feitas sobre. Conforme afirma Savi (2011), o design instrucional consiste em uma série de passos para identificar necessidades de aprendizagem e alinhar elementos de conteúdo para atender a essas necessidades.

Contudo, vários autores, entre eles Aleven et al. (2010), Echeverría et al. (2011), Villalta et al. (2011), Savi (2011), entre outros, acrescentam aos princípios e processos do design instrucional a utilização da taxonomia de Bloom para a definição dos objetivos educacionais do jogo. Também verificou-se com base nos estudos Gomes (2001), e Campos e Macedo (2011) a utilização dos critérios de mediação de Feuerstein para a criação de bons jogos educativos digitais.

Portanto, para a criação dos jogos educativos digitais, sugere-se utilizar ambas as teorias, pois elas podem ser colaborativas e complementares para o processo de ensino e aprendizagem.

\section{Proposta lúdica para a criação de jogos educativos digitais}

Referente à proposta lúdica, conforme síntese apresentada no quadro 3, para a criação de jogos educativos, propõem-se o uso de quatro elementos principais, que são: Mecânica; estética; história/narrativa; e tecnologia.

A seleção destes elementos se baseia nos trabalhos de Hunicke et al. 2004, Schell (2008), Aleven et al. (2010), Echeverría et al. (2011), Villalta et al. (2011), entre outros. Sendo que, segundo Echeverría et al. (2011), a dimensão lúdica determina os elementos de um jogo digital qualquer, respeitando as restrições impostas pela dimensão educativa.

Mecânica - A criação da mecânica do jogo está vinculada à determinação das regras, normas, procedimentos, metodologias, colaboração, materiais etc. $\mathrm{O}$ desenvolvimento da mecânica deve respeitar os objetivos 


\section{$16^{\circ}$ \\ ERGODESIGN USIHC CINAHPA}

pedagógicos definidos para o jogo (HUNICKE et al., 2004; SCHELL, 2008; ALEVEN et al., 2010; ECHEVERRÍA et al., 2011; VILLALTA et al., 2011). A mecânica aplicada no jogo, segundo Hunicke et al. (2004) e Aleven et al (2010), determina a dinâmica dos comportamentos dos jogadores. Segundo Villata (2011), a mecânica do jogo está ligada aos objetivos da aprendizagem. O conteúdo curricular deve ser incorporado à mecânica de funcionamento do jogo, de tal forma que o sucesso deste é condicional para a compreensão de seu conteúdo.

Estética - A estética conforme Hunicke et al. (2004) e Aleven et al (2010) visa capturar a experiência subjetiva do jogador, a resposta emocional ou o prazer que o jogo evoca. Os autores descrevem uma taxonomia de oito elementos estéticos, que inclui: sensação, fantasia, narrativa, desafio, camaradagem, descoberta, expressão e submissão. A estética do jogo, segundo Echeverría et al. (2011), descreve a aparência do jogo (design gráfico, cores, avatares, objetos etc.) e sons (música, efeitos sonoros etc.). Busca definir o tom geral do jogo, o que afetará os sentimentos e experiências de um jogador ao jogar.

História/Narrativa - A narrativa é a linguagem utilizada na história dos jogos. A narrativa e a história possuem a função de determinar o roteiro de eventos, a progressão e a dinâmica do jogo. Elas devem proporcionar relevância e imersão para o jogador, motivando-o a jogar. A história, conforme Echeverría et al (2011), descreve a sequência de eventos que se desenrolam durante um jogo. Ela pode ser muito simples e linear, ou altamente complexa e ramificada. Já Villata et al. (2011) entendem que a progressão do jogo deve ter uma narrativa clara, que permita a imersão dos participantes na história do jogo.

Tecnologia - A tecnologia é apresentada por Echeverría et al. (2011) como elemento lúdico, no que se refere aos tipos de materiais e interações que tornam possível o jogo de ser jogado. Segundo o autor, ela permite que o $16^{\circ}$ Ergodesign - Congresso Internacional de Ergonomia e Usabilidade de Interfaces Humano Tecnológica: Produto, Informações Ambientes Construídos e Transporte

$16^{\circ}$ USIHC - Congresso Internacional de Ergonomia e Usabilidade de Interfaces Humano Computador

CINAHPA | 2017 - Congresso Internacional de Ambientes Hipermídia para Aprendizagem.

jogo possa fazer certas coisas, enquanto proíbe de fazer outras. A tecnologia, numa visão ampla, é abordada por todos os autores pesquisados. Ela influencia todos os elementos dos jogos. Existem vários tipos de tecnologias que podem servir de suporte aos jogos, tais como: consoles, computadores desktoop ou notebooks, celulares, tablets etc.

A proposta lúdica para a criação dos jogos educativos digitais visa apresentar os elementos lúdicos a serem considerados no desenvolvimento dos jogos.

\section{Considerações finais}

Neste trabalho, contatou-se que a utilização dos jogos educativos digitais agrega valor, como estratégia pedagógica para o ensino e aprendizagem de conteúdos em sala de aula.

Também se verificou que outros elementos relevantes para o processo de criação de jogos podem ser incorporados ao design instrucional, no intuito que os desenvolvedores tenham melhor clareza dos elementos pedagógicos e lúdicos a serem concebidos no design do jogo.

Referente à estrutura pedagógica, a presente proposta, demonstra que a utilização da taxionomia de Bloom revisada apoia a definição dos objetivos educacionais do jogo. Assim como, os critérios de mediação de Feuerstein possibilita a concepção de estratégias pedagógicas que facilitem a mediação do processo de ensino e aprendizagem em sala de aula. Já as recomendações dos elementos lúdicos (mecânica, estética, história/narrativa e tecnologia) a serem incorporados nos jogos, ajuda os desenvolvedores, ligados aos processos de design instrucional, a criarem jogos que motivem os educandos a jogar.

\section{BIBLIOGRAFIA}

ALEVEN, V. et al. Toward a framework for the analysis and design of educational games. In: IEEE - INTERNATIONAL 


\section{$16^{\circ}$ \\ ERGODESIGN USIHC CINAHPA}

\author{
CONFERENCE ON DIGITAL GAME AND \\ INTELLIGENT TOY ENHANCED \\ LEARNING. Proceedings, p. 69-76. 2010.
}

BALASUBRAMANIAN, N.; WILSON, B. G. Games and simulations. In: SOCIETY FOR INFORMATION TECHNOLOGY AND TEACHER EDUCATION

INTERNATIONAL CONFERENCE, 2006.

Proceedings. v. 1, 2006. Disponível em: $<$ http://site.aace.org/pubs/foresite/GamesAndSi mulations1.pdf>. Acesso em: 08 jan. 2012.

CAMPOS, M. C. R. M.; MACEDO, L. Desenvolvimento da função mediadora do professor em oficinas de jogos. Revista Semestral da Associação Brasileira de Psicologia Escolar e Educacional. São Paulo: v. 15, n 2, p. 211-220. Jul/Dez., 2011.

ECHEVERRÍA, A. et al. A framework for the design and integration of collaborative classroom games. Computers \& Education, v. 57, p. 1127-1136, 2011. Disponível em: <www.elsevier.com/locate/compedu $>$. Acesso em: 10 mar. 2012.

GOMES, C. M. A. Em busca de um modelo psico-educativo para a avaliação de softwares educacionais. Dissertação (Mestrado em Engenharia de Produção) Programa de Pós-Graduação em Engenharia de Produção, Universidade Federal de Santa Catarina, Florianópolis, 2001. Disponível em: <http://aspro02.npd.ufsc.br/pergamum/bibliote ca/index.php?resolution $2=$

024_1\&tipo_pesquisa=\#posicao_dados_acervo >. Acesso em: 15 fev. 2012.

HUNICKE, R. et al. MDA: A formal approach to game design and ame research. In: FU, D.; HENKE, S.; ORKIN, J. (Eds.). Challenges in game artificial intelligence. Papers from the 2004 AAAI WORKSHOP TECHNICAL REPORT WS-04-04. Menlo Park, CA: The AAAI Press, p. 1-5, 2004.

JAPPUR, Rafael F. Modelo conceitual para a criação, aplicação e avaliação de jogos $16^{\circ}$ Ergodesign - Congresso Internacional de Ergonomia e Usabilidade de Interfaces Humano Tecnológica: Produto, Informações Ambientes Construídos e Transporte

$16^{\circ}$ USIHC - Congresso Internacional de Ergonomia e Usabilidade de Interfaces Humano Computador

CINAHPA | 2017 - Congresso Internacional de Ambientes Hipermídia para Aprendizagem.

educativos digitais. Tese (Doutorado em Engenharia e Gestão do Conhecimento) Programa de Pós-Graduação em Engenharia e Gestão do Conhecimento, Universidade Federal de Santa Catarina, Florianópolis, 2014. Disponível em: <

http://btd.egc.ufsc.br/?p=1896>. Acesso em: 15 jan. 2017.

KIRRIEMUIR, J; MCFARLANE, A. Literature review in games and learning. Bristol: Futurelab, 2004. Disponível em: <www.mendeley.com/research/literaturereview-in-games-and-learning/>. Acesso em: 05 jan. 2012.

MAYER, Richard E. A taxonomy for computer-based assessment of problem solving. Computers in Human Behavior, v. 18, p. 623-632, 2002.

MORENO-GER, P. et. al. Educational game design for online education. P. Computers in Human Behavior, v. 24, p. 2530-2540, 2008.

PRENSKY, M. Digital game-based learning. McGraw-Hill, New York, 2001.

SAVI, Rafael. Avaliação de jogos voltados para a disseminação do conhecimento. Tese (Doutorado em Engenharia e Gestão do Conhecimento) - Programa de Pós-Graduação em Engenharia e Gestão do Conhecimento, Universidade Federal de Santa Catarina, Florianópolis, 2011. Disponível em: $\langle$ http://btd.egc.ufsc.br/?p=1038>. Acesso em: 15 jan. 2012.

SCHELL, J. The art of game design: a book of lenses. Morgan Kaufmann. 2008.

VILLALTA. M. et al. Design guidelines for classroom multiplayer presential games (CMPG). Computers \& Education, v. 57, p. 2039-2053, 2011. Disponível em: <www.elsevier.com/locate/compedu $>$. Acesso em: 10 de mar. de 2012.

\section{Realização: $\prod_{\substack{\text { țU士 } \\ \text { veta }}}$}

